\title{
The acute effects of aerobic exercise on sensorimotor adaptation in chronic stroke
}

\author{
Christopher P. Mackay ${ }^{\mathrm{a}, *}$, Sandra G. Brauer ${ }^{\mathrm{a}}$, Suzanne S. Kuys ${ }^{\mathrm{b}}$, \\ Mia A. Schaumberg ${ }^{\mathrm{c}, \mathrm{d}, \mathrm{e}}$ and Li-Ann Leow ${ }^{\mathrm{e}, \mathrm{f}}$ \\ ${ }^{\mathrm{a}}$ The University of Queensland, School of Health and Rehabilitation Sciences, Brisbane, Queensland, Australia \\ ${ }^{\mathrm{b}}$ Australian Catholic University, School of Allied Health, Brisbane, Queensland, Australia \\ ${ }^{\mathrm{c}}$ University of the Sunshine Coast, School of Health and Sport Sciences, Maroochydore, Queensland, Australia \\ ${ }^{\mathrm{d}}$ Sunshine Coast Health Institute, Birtinya, Queensland, Australia \\ ${ }^{\mathrm{e}}$ The University of Queensland, School of Human Movement and Nutrition Science, Brisbane, Queensland, \\ Australia \\ ${ }^{\mathrm{f}}$ The University of Queensland, School of Psychology, Brisbane, Queensland, Australia
}

\begin{abstract}
.
Background: Sensorimotor adaptation, or the capacity to adapt movement to changes in the moving body or environment, is a form of motor learning that is important for functional independence (e.g., regaining stability after slips or trips). Aerobic exercise can acutely improve many forms of motor learning in healthy adults. It is not known, however, whether acute aerobic exercise has similar positive effects on sensorimotor adaptation in stroke survivors as it does in healthy individuals.

Objective: The aim of this study was to determine whether acute aerobic exercise promotes sensorimotor adaptation in people post stroke.

Methods: A single-blinded crossover study. Participants attended two separate sessions, completing an aerobic exercise intervention in one session and a resting control condition in the other session. Sensorimotor adaptation was assessed before and after each session, as was brain derived neurotrophic factor. Twenty participants with chronic stroke completed treadmill exercise at moderate to high intensity for 30 minutes.

Results: Acute aerobic exercise in chronic stroke survivors significantly increased sensorimotor adaptation from pre to post treadmill intervention. The 30-minute treadmill intervention resulted in an averaged $2.99 \mathrm{ng} / \mathrm{ml}$ increase in BDNF levels $($ BDNF pre-treadmill $=22.31+/-2.85 \mathrm{ng} / \mathrm{ml}$, post-treadmill was $=25.31+/-2.46 \mathrm{pg} / \mathrm{ml} ; \mathrm{t}(16)=2.146, p=0.048$, cohen's $d=0.521$, moderate effect size).

Conclusions: These results indicate a potential role for aerobic exercise to promote the recovery of sensorimotor function in chronic stroke survivors.
\end{abstract}

Keywords: Sensorimotor adaptation, aerobic exercise, stroke

\section{Introduction}

Change in the brain due to neuroplasticity is a foundation principle underpinning the rehabilitation of motor function following stroke. Plastic effects of acute aerobic exercise on the brain have been

\footnotetext{
${ }^{*}$ Corresponding author: Christopher P. Mackay, School of Health and Rehabilitation Sciences, The University of Queensland, Brisbane, Queensland, Australia. Tel.: +61 73365 2317; Fax: +61 73346 8789; E-mail: c.mackay@uq.edu.au.
}

demonstrated in many domains (Chang et al., 2012; Thomas et al., 2016; Brown \& Bray, 2018), including in motor learning (Roig et al., 2012; Mang et al., 2014; Ferrer-Uris et al., 2017; Stavrinos \& Coxon, 2017; Angulo-Barroso et al., 2019; Neva et al., 2019). The potential for exercise to augment motor learning in stroke patients has particular clinical utility in improving motor rehabilitation (Kitago \& Krakauer, 2013), as current rehabilitation practices result in relatively modest improvements in residual 
motor deficits experienced by many stroke survivors (Stinear et al., 2020). Better understanding of how exercise might improve motor learning in stroke will inform judicious design of how to incorporate exercise into rehabilitation interventions targeting persistent residual motor deficits. To this end, two studies have tested the acute effects of exercise on motor learning in stroke (Nepveu et al., 2017; Charalambous et al., 2018). Although stroke survivors have reduced exercise capacity even 6 months poststroke (Mackay-Lyons \& Makrides, 2004), patients can still benefit from exercise interventions (Globas et al., 2012). Nepveu et al. (2017) had chronic stroke survivors either rest or complete high-intensity interval training immediately after a skill acquisition task where survivors adapted grip forces to control an on-screen cursor. Fifteen minutes of high intensity interval training improved performance when participants re-encountered the same task 24 hours later. In contrast, Charalambous et al. (2018) found no benefit of $\approx 5$ mins exercise on split-belt treadmill adaptation, a motor learning task requiring gait adaptation to different treadmill belt speeds, possibly due to the reduced exercise duration in this study.

A recent systematic review suggests that effects of exercise on motor learning depend on when exercise is administered, exercise dose (intensity and/or duration), and the nature of the motor learning task (Wanner et al., 2020). More intense exercise administered immediately after motor learning tended to have most prominent effects on the consolidation but not the encoding of motor learning. The review also suggests that exercise might particularly benefit a form of motor learning termed sensorimotor adaptation: although there were fewer published studies on exercise in sensorimotor adaptation, effect sizes were overall larger with sensorimotor adaptation than with other forms of motor learning (Wanner et al., 2020). An additional benefit of the sensorimotor adaptation paradigm is its long history of study: there are clear theories on its mechanisms and a rich body of knowledge on its characteristics in healthy and clinical populations (Morehead \& de Xivry, 2021), including in stroke (e.g., Mutha et al., 2011). In sensorimotor adaptation, the sensory feedback of a movement is experimentally perturbed, leading to a discrepancy between the predicted sensory outcome and the actual sensory outcome (sensory prediction error) (Tseng et al., 2007). A perturbation can be created by rotating the visual feedback of a hand movement in a $30^{\circ}$ clockwise direction during a target reaching task. Perturbations lead to discrepancies between the pre- dicted sensory feedback about the movement and the actual sensory feedback received (i.e., sensory prediction errors) (Jordan, 1992). With repeated reaches, this sensory prediction error triggers an updating of the motor command to reduce the sensory prediction error, and this learning occurs in an implicit, automatic way. Perturbations also often lead to discrepancies between the predicted task outcomes (e.g., hit the target) and the actual task outcomes (e.g., fail to hit the target), here termed task errors (Welch, 1969; Schaefer et al., 2012; Leow et al., 2018; Kim et al., 2019). Such task errors are thought to promote the use of explicit strategies, to obtain desired task outcomes. Quantifying how quickly individuals return sensory prediction errors and task errors to pre-perturbation levels measures the brain's ability to learn to adapt to altered sensory feedback via implicit and explicit learning mechanisms, and may indicate the neuroplastic capacity of the individual (Seidler \& Carson, 2017).

Here, we designed a study examining the acute effect of 30 minutes of moderate to high intensity aerobic exercise on sensorimotor adaptation with goal-directed reaching of the upper limbs in chronic stroke patients. The aim of this study was to determine if a single bout of moderate to high intensity aerobic exercise performed by people following stroke improved sensorimotor adaptation compared to a control period of rest. We hypothesised that acute aerobic exercise would improve the sensorimotor adaptation of individuals post stroke.

\section{Methods}

\subsection{Participants}

Twenty people with chronic stroke (Table 1) participated in the study (aged $61 \pm 13$ years; $76 \%$ male). To be included in the study, participants had to be diagnosed with a stroke at least 3 months prior (Bernhardt et al., 2017), be able to walk with or without an aid for at least 10 metres and be able to understand three-stage commands. Individuals were excluded if

Table 1

Participant characteristics $(n=20)$

\begin{tabular}{lc}
\hline Average age & 60 years \pm 14 \\
Time since stroke & 3 years, 11 months \\
Male participants & $76 \%$ \\
10 Metre Walk Time & 8.5 seconds \\
Dominant hand used for reach & $17 / 20$ or $85 \%$
\end{tabular}


they were unable to walk independently prior to the current stroke, had co-morbidities that might limit their walking (such as arthritis), had an unstable cardiac status, or were unable to understand instructions or provide informed consent. Participants who had a non-functional upper limb post stroke (that was also their dominant limb) were not excluded from the study, but were asked to use their other upper limb to complete the sensorimotor task.

A single bout of exercise induced changes in brain-derived neurotrophic factor (BDNF) levels in a sample of 20 people with multiple sclerosis (Schulz et al., 2004; Castellano \& White, 2008). Both studies found a change of BDNF levels of $5 \mathrm{ng} / \mathrm{ml}$ from pre to post, with a maximum SD of $5 \mathrm{ng} / \mathrm{ml}$. We estimated that a sample size of 18 is required to detect a difference of $5+/-5 \mathrm{ng} / \mathrm{ml}$ at a two sided 0.05 significance level with a power of $80 \%$. We note however that the true effect size might be less than this due to publication bias (Szuhany et al., 2015), as it remains unclear what the minimum sample size is to detect exercise-induced changes in BDNF in a chronic stroke population.

Information collected to describe the sample participants included date, location and type of stroke, age, sex, medical co-morbidities and current medications. Ethical approval for the study was obtained through the local human research ethics committee. All participants provided written informed consent in accordance with the Declaration of Helsinki.

\subsection{Experimental design}

In a cross-over design, each participant was pseudo-randomised to complete an intervention condition (Treadmill) or control condition (Rest) first, returning at least one week later (washout period) to complete the alternate condition. A minimum washout period of one week was used to allow for any physiological changes due to exercise to return to resting levels, but to minimise any stroke recovery-associated changes to function. Participants were asked to refrain from exercising in the 24 hours prior to their visit. At each visit, participants completed the sensorimotor adaptation task on two occasions, pre and post intervention, resulting in four assessment timepoints (PreControl, PostControl and PreTreadmill, PostTreadmill). The post intervention assessment aimed to commence within 15 minutes of completing the treadmill or rest condition.
Table 2

Exercise performance data $(n=20)$

\begin{tabular}{lc}
\hline & Mean \& St. Dev \\
\hline TREADMILL DATA & \\
Speed Maximum (km/hr) & $3.94 \pm 1.54$ \\
Time (mins) & $28.58 \pm 3.46$ \\
Distance (metres) & $1761.2 \pm 660.8$ \\
Maximum predicted HR (bpm) & $157 \pm 16$ \\
HR Max achieved (bpm) & $122 \pm 19$ \\
$\%$ of Max HR & $78.3 \pm 13.6$ \\
Modified Borg Maximum & $13 \pm 1$ \\
\hline
\end{tabular}

Legend: HR - Heart Rate, bpm - beats per minute.

\subsection{Intervention}

The intervention condition (Table 2) consisted of a single session of moderate-high intensity aerobic exercise (65\% of heart rate reserve) for 30 minutes walking on a standard treadmill (Landice L7 treadmill), with arms placed comfortably by participants on the rail (in front) or swinging freely. Information describing the specific features of the training session (e.g. heart rate, blood pressure response, treadmill speed, total distance walked) were recorded to monitor the response to exercise and adherence to the exercise protocol. The single treadmill session included a progressive increase in intensity (usually increased speed, or gradient) to reach the target heart rate (approx. 5 mins) as well as a cooling down period (approx. 5 mins) to allow for participants to return towards resting levels for vital observations. The warm-up and cool-down were included as part of the total 30 minutes of walking exercise. Target heart rates were calculated using the Karvonen method (Lawrence, 2006) with levels adjusted for those taking heart rate lowering medications (i.e., beta blockers), following methods previously published in post stroke populations (MacKay-Lyons \& Makrides, 2002; Kuys et al., 2011). Heart rate was measured using a chest strap monitor (polar-electro) and monitored by a research assistant who facilitated changes in treadmill parameters to enable participants to reach their target. Participants were asked to self-rate their intensity of exercise every 10 minutes verbally using BORG's 6-20 scale rating of perceived exertion (Borg, 1982). Participants were instructed to walk at a pace that resulted in a rating between 11 (fairly light) and 14 (somewhat hard) on the scale. The control condition involved an equivalent time period (30 minutes) of seated resting where participants were provided with an education session about the impact and effects of stroke by the same research assistant. 
A.

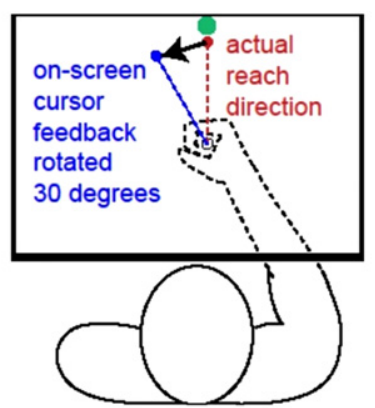

C.

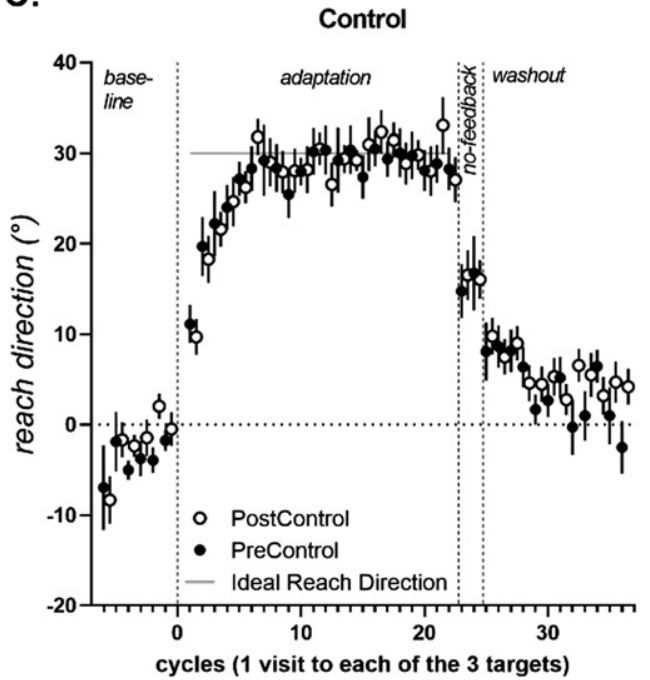

B.

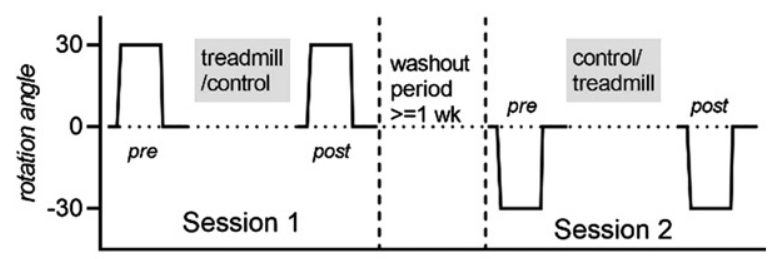

D.

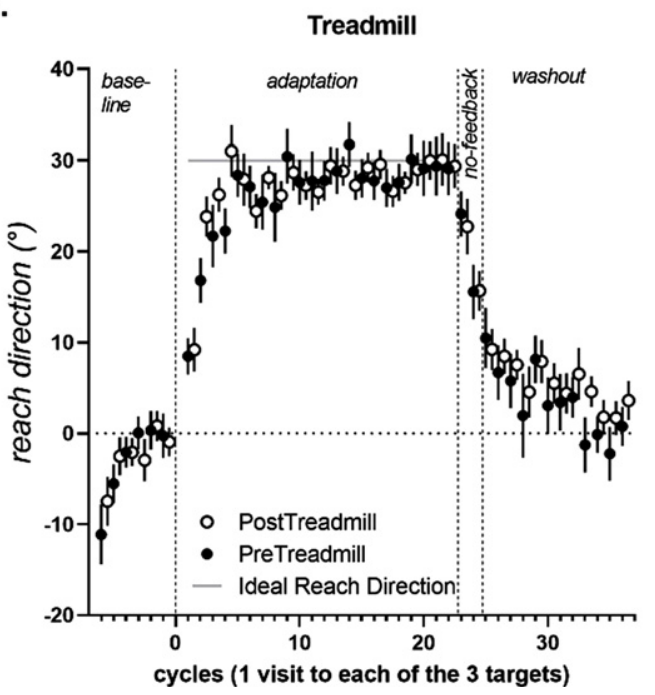

Fig. 1. A. Experimental set-up. Vision of the arm is occluded as participants make reaching movements on a digitizing tablet placed under a stand: cursor feedback of the hand is shown on a monitor screen placed horizontally on the stand. B. All participants completed Session 1 with a 30 degree clockwise rotation of cursor feedback, and the ideal reach direction was 30 degrees clockwise. On Session 2, participants encountered a 30 degree counter clockwise rotation, and the ideal reach direction was 30 degrees clockwise. C\&D. Mean reach direction $(+/-$ standard errors of the mean) for each cycle ( 1 cycle $=1$ visit to each of the 3 targets $)$, for the control and treadmill conditions. For ease of comparison, datasets in which participants experienced a clockwise rotation (and had a -30 ideal reach direction) were sign-flipped for this figure, such that reaches that are close to +30 represent better adaptation for all datasets. Data from the Post conditions are offset by 0.5 to avoid being obscured by data from the Pre conditions.

\subsection{Sensorimotor adaptation task}

\subsubsection{Apparatus and setup (Fig. 1)}

Participants were seated in front of a desk (approximately $50 \mathrm{~cm}$ from their coronal plane) and asked to move a digitising pen $(15.95 \mathrm{~cm}$ long, $1.4 \mathrm{~cm}$ wide, $17 \mathrm{~g}$ ) on a digitizing tablet (WACOM Intuos4 PTK 1240 , size: $19.2 \times 12$ in., resolution $=0.25 \mathrm{~mm}$ ) from an origin to a target point. The pen's position on the tablet (XY coordinates) was sampled at $100 \mathrm{~Hz}$ and displayed in real time as a circular cursor with a 5-pixel radius $(1.25 \mathrm{~mm})$ on a horizontally placed computer monitor. Direct vision of the hand was prevented by placing the tablet and the hand directly beneath an opaque stand, with the horizontal monitor placed atop the stand.

\subsubsection{Task instructions}

Participants first received task instructions to move an on-screen cursor from the start to the target, in a straight line, in a single movement, as quickly and as accurately as possible. Further, participants were instructed that the feedback of the movement would be changed from time-to-time, and that participants were to change their movement in response to this change of feedback, whilst keeping movements as straight as possible.

In each trial, the participants' task was to move from the origin location through the target location as quickly and accurately as possible using their dominant upper limb. Targets were presented in one of three locations $(210,225$ or 240 degrees from the right horizontal plane) in random order. These target 
directions were selected such that target-reaching movements involved the horizontal adductors of the shoulder joint. After moving through the target, or past the target, a high-pitched tone sounded to indicate trial completion. Following completion of a trial the origin location was re-displayed immediately and participant directed to repeat task. If the participant did not complete a successful trial, they were directed to return to the origin location and repeat the trial.

First, participants encountered 18 baseline trials under normal (correct) feedback with no rotation. Participants then immediately completed 66 adaptation trials, where the visual feedback on the display monitor was perturbed by rotating it 30 degrees in a clockwise direction (1st testing day) or counter-clockwise direction (2nd testing day). After the adaptation block, to notify participants that the perturbation had been removed, a popup dialog box appeared with the statement "In the next few trials, the disturbance that the computer applied will be removed. Please aim straight to the target." The instructions on-screen were read out by the experimenter to ensure it was understood. This was immediately followed by 6 nofeedback trials. Finally, participants completed 36 washout trials under normal cursor feedback conditions (i.e., no cursor rotation), to return behaviour to an unadapted state.

\subsection{Sensorimotor adaptation data processing and analysis}

Custom scripts written in LabVIEW scored reach directions, which were quantified at the 15 th data point (150 milliseconds into the reach), as online movement corrections typically occur after $150 \mathrm{~ms}$ into a reach. Trials with reach direction outside a 120 degree range of the target (60 degrees on either side of the target) were discarded as outliers (Leow et al., 2017). Trials were binned into cycles of one visit to each of the three targets. The dependent variable was percent adaptation (Krakauer et al., 2005), which quantified reach directions in every cycle relative to the ideal reach direction by calculating reach directions as a percentage of ideal reach directions resulting from perfect adaptation performance. Ideal reach direction was 30 degree clockwise for a 30 degree counter-clockwise rotation, and 30 degrees counter-clockwise for a 30 degree clockwise rotation.

Percent adaptation $=100 \% \times($ reach direction $) /$ (ideal reach direction)
As observed previously (Krakauer et al., 2005), there was a rapid error reduction phase of reaching where a majority of learning occurred followed by a slower rate of adaptation. Here, as the targets were spaced close together, rapid error reduction occurred in trials 1-9 (i.e., the first three cycles), similar to recent work (Huberdeau et al., 2015). At completion of the fourth cycle, adaptation was greater than $70 \%$ both before and after the treadmill and control conditions. We thus selected the first three cycles to quantify rapid error reduction.

Individual differences in reach directions at baseline can affect measures of adaptation (Ghilardi et al., 1995). Previous methods of accounting for this by subtracting pre-perturbation behaviour from postperturbation behaviour is more sensitive to noisy baselines and risk of Type 2 error (Vickers, 2001). To account for pre-perturbation baseline biases, we entered percent adaptation averaged from the final three cycles before rotation onset (i.e., percent adaptation in the last five baseline cycles) as covariates in all of our analysis of covariance analyses.

After the adaptation trials, there were two cycles where no feedback was provided to the participant. That is, unlike all previous trials where participants could follow a tracking cursor with their vision, the participants received no real time feedback about where they were reaching. The first no-feedback cycle was taken as a measure of implicit learning, similar to previous work (Heuer \& Hegele, 2008).

Our outcome measures of interest were (1) adaptation performance (2) implicit aftereffects, quantified as reaches that remained adapted despite notification of perturbation removal in the no-feedback block and (3) explicit learning, estimated as the volitional disengagement of adapted behaviour after receiving notification of perturbation removal (i.e., the change in percent adaptation from the mean of the last three adaptation cycles to the first no-feedback cycle after receiving notification that the perturbation was gone), and (4) de-adaptation performance. To evaluate these measures, we ran ANCOVAs with the betweensubjects factor Intervention Order (Control First, Treadmill First) and the within-subjects factors: Intervention (Control, Treadmill), Time (Pre-Intervention, Post-Intervention) and (where applicable) Cycles (cycles 1-3), with pre-rotation biases as covariates of no interest (estimated from mean percent adaptation in the last three baseline cycles). Where appropriate, Greenhouse-Geisser corrections were applied. Alpha was set at 0.05. SPSS v24.0 was used for statistical analyses. 
A.

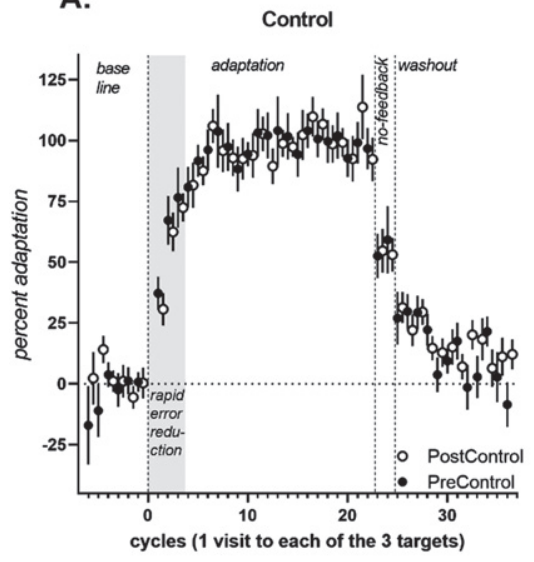

B.

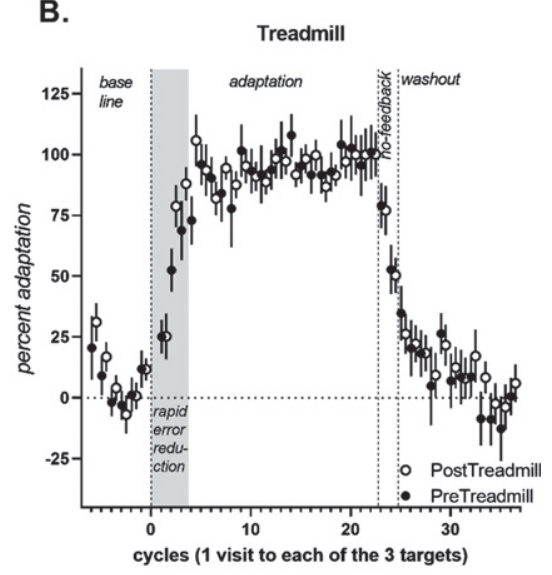

rapid error reduction phase

C.

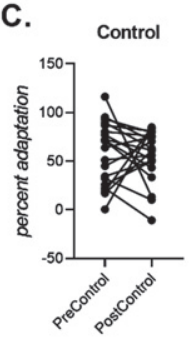

D. Control

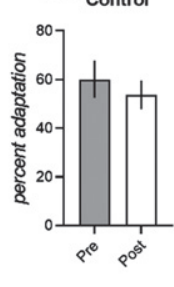

E.

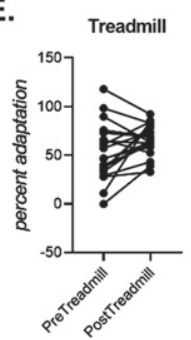

F. Treadmill

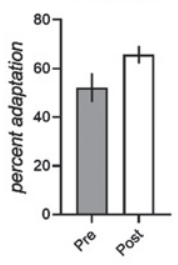

Fig. 2. Percent adaptation of participants during the adaptation (cycles 1-22), no-feedback (cycles 23-24) and washout (cycles 25-36) trials for the control condition (A) and the exercise condition (B). Note that data from the Post conditions are offset by 0.5 to avoid being obscured by data from the Pre conditions. There was a significant interaction between the control and exercise condition from pre to post in the rapid error reduction phase (shaded region, i.e., first 3 adaptation cycles). Individual participant data in the rapid error reduction phase is shown for the control condition (C) and the treadmill condition (E). Covariate adjusted mean percent adaptation $+/$ - standard error of the mean for the rapid error reduction phase is shown for the control (D) and treadmill (F) conditions

\subsection{BDNF analysis}

Blood was collected in $9 \mathrm{~mL}$ serum separation tubes that contained gel and clot activator, allowed to clot at room temperature for 20-30 minutes then centrifuged at $3000 \times \mathrm{g}$ for 10 minutes at $4^{\circ} \mathrm{C}$ and stored at $-80^{\circ} \mathrm{C}$ for later analysis. Serum BDNF concentrations were evaluated via an enzyme linked immunosorbent assay ( $R \& D$ systems Quantikine ELISA - Total BDNF kit) according to the protocol provided by the manufacturer.

\section{Results}

\subsection{Reaching with rotated feedback (adaptation trials) - Figure 2}

During the rapid error reduction phase of the adaptation trials (cycles 1-3), a significant Intervention $\times$ Time interaction was observed $[\mathrm{F}(1,13)=$
$6.399, p=0.027$, partial eta squared $=0.346]$. Followup ANCOVAs were run separately for the Treadmill and Control interventions. Percent adaptation increased pre-to-post in the Treadmill intervention [Fig. 2B, significant main effect of Time, $F(1,15)=$ $6.241, p=0.025$, partial eta-squared $=0.247$, PreTread $52.5 \pm 6.2 \%[39.1,66.0]$ vs. PostTread 65.7 \pm $3.8 \%)[57.4,74.0]$, but not for the Control intervention [Fig. 2A, non-significant main effect of Time, $\mathrm{F}(1,15)=1.138, p=0.303$, partial eta-squared $=0.07$, PreControl $62.8 \pm 5.9 \%$ [49.9\%, 75.9\%] vs. PostControl $53.9 \pm 6.1 \%[40.5 \%, 67.5 \%]]$.

\subsection{Implicit aftereffects and Explicit learning - Figure 3}

Implicit learning (Fig. 3A), measured as percent adaptation in the first no-feedback cycle after receiving notification of the perturbation removal (Heuer 
A.

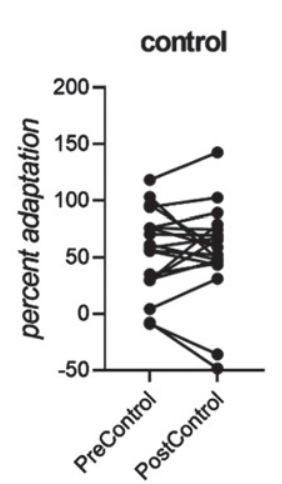

treadmill

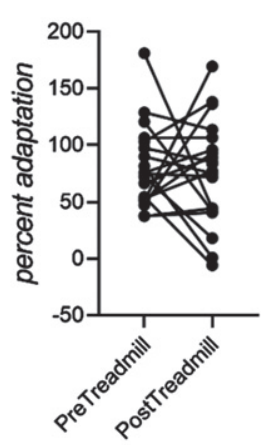

B. explicit learning

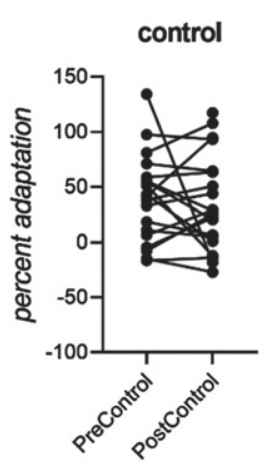

treadmill

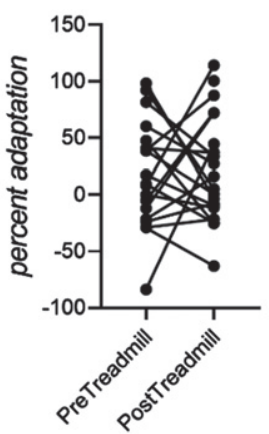

Fig. 3. A. Individual participant data for implicit learning, measured as percent adaptation in the first no-feedback cycle (i.e., movements that remained adapted despite being notified that the perturbation had been removed). B. Individual participant data for explicit learning, measured as the change in percent adaptation upon notification of the perturbation removal). Implicit and explicit learning did not differ significantly from pre- to post-control and/or pre-to-post intervention.

\& Hegele, 2008), was larger overall in the treadmill intervention conditions than the control intervention conditions, but did not differ significantly from preto post- between control and treadmill conditions, as the Intervention $\times$ Time interaction was not significant, $(p>0.05)$. The main effect of treadmill and the main effect of Time was also not significant.

All conditions showed a clear change in reach directions after receiving instructions that the perturbation had been removed (see Fig. 2), indicating a role for explicit learning in this sensorimotor adaptation task. Explicit learning, as assessed here at the end of each adaptation block, did not differ reliably between before and after the control and treadmill interventions (all main effects of Intervention and Time, and all interactions with Intervention and Time $p>0.05$ ).

\subsection{Reaching with normal feedback (de-adaptation trials)}

Pre- and post-intervention de-adaptation did not differ significantly between Control and Treadmill intervention conditions, [non-significant main effect of Intervention, non-significant $\times$ Time interaction, $\mathrm{F}(1,12)=0.4, p=0.538$, partial $\eta$-squared $=0.03$ ). Participants in both intervention conditions returned to a normal level of baseline reaching, as expected.

\subsection{Effect of exercise on BDNF}

Although the primary goal of the study was not to examine effects of exercise on BDNF, and thus sample size was likely underpowered to detect exercise-induced changes in BDNF, we ran exploratory analyses to examine if the treadmill exercise intervention changed BDNF levels. The 30-minute treadmill intervention resulted in an averaged $2.99 \mathrm{ng} /$ $\mathrm{ml}$ increase in BDNF levels (BDNF pre-treadmill $=$ $22.31+/-2.85 \mathrm{ng} / \mathrm{ml}$, post-treadmill was $=25.31+/$ $-2.46 \mathrm{pg} / \mathrm{ml} ; \mathrm{t}(16)=2.146, p=0.048$, cohen's $d=$ 0.521 , moderate effect size). The control condition resulted in an averaged $1.60 \mathrm{pg} / \mathrm{ml}$, non-statistically significant increase in BDNF from pre- to post $\mathrm{t}(16)=1.400, p=0.181$, cohen's $d=0.340$, small effect size). To explore the relationship between treadmill-induced BDNF change with treadmillinduced performance improvements early in the adaptation block, we first quantified (1) BDNF change pre-to-post treadmill = post-treadmill BDNF - pre-treadmill BDNF, (2) performance change preto-post treadmill $=$ post treadmill percent adaptation mean-averaged from the first 3 adaptation cycles pre treadmill percent adaptation mean-averaged from the first 3 adaptation cycles; and then ran pearson's correlations between these two variables. Treadmillinduced BDNF change showed a non-significant positive correlation with treadmill-induced improvements in early adaptation $(r=0.340, p=0.198)$.

\section{Discussion}

This study found that a single bout of moderate to high intensity aerobic exercise was sufficient 
to increase sensorimotor adaptation performance of chronic stroke survivors. The improvement in reaching performance (observed as a faster adaptation post-intervention compared to pre-intervention, often termed savings) was observed during the rapid error reduction phase of the trials after the treadmill exercise, but not after the rest condition. Thus, the present study showed that a single session of moderate to high intensity aerobic exercise increased capacity to improve sensorimotor adaptation in chronic stroke survivors.

Accelerated improvement in adaptation performance (savings) post-exercise was specifically seen in the rapid error reduction phase of adaptation. Participants demonstrated increased ability to readapt to the imposed rotation in the first nine trials following the exercise compared to the control condition. Such an improvement in performance following exercise may represent an acute change in the brain leading to an enhanced internal environment that could facilitate adaptation. Although exercise-induced improvements in motor skill learning have previously been shown in stroke patients (Nepveu et al., 2017), this is the first study to our knowledge to demonstrate an improvement in sensorimotor adaptation following moderate-high intensity exercise in a stroke population. Our findings corroborates other findings of improved sensorimotor adaptation (i.e., increased savings) with exercise in young healthy adults (Ferrer-Uris et al., 2017; Neva et al., 2019) and in children (Angulo-Barroso et al., 2019). We did not detect an effect of exercise on the implicit aftereffect measured nor in explicit learning when measured at the end of the adaptation block. This might have resulted from exercise having a selective effect early and not late in the adaptation block. We cannot rule out an effect of exercise on implicit and explicit learning, as we did not measure implicit and explicit learning early during adaptation. We speculate that faster re-adaptation with exercise shown here in chronic stroke patients results from similar mechanisms as in neurologically intact individuals (Ferrer-Uris et al., 2017; Angulo-Barroso et al., 2019; Neva et al., 2019). One open question is whether chronic stroke results in selective deficits in savings, which renders stroke patients more sensitive to beneficial effects of exercise on savings: to the best of our knowledge, the majority of previous reach adaptation studies in stroke patients have not explicitly examined savings (Schaefer et al., 2009; Mutha et al., 2011). These results of exercise-induced benefits to motor learning stands in contrast with other work by Charalambous et al. $(2018,2019)$ who found no effects of exercise on consolidation in locomotor adaptation in stroke patients (Charalambous et al., 2018) and in healthy controls (Charalambous et al., 2019). Several methodological differences might have led to this discrepancy. First, although locomotor adaptation is susceptible to effects of explicit learning, people do not appear to actively engage explicit learning in locomotor adaptation (Roemmich et al., 2016). In contrast, behaviour in the type of reach adaptation paradigm used here has a large contribution from explicit learning (Taylor et al., 2014). Here, exercise had a selective influence on improved re-adaptation to a previously encountered perturbation (i.e., savings), which often has a contribution from explicit learning (Morehead et al., 2015). Exercise might have modulated adaptation by modulating explicit learning processes, which might be more prominent in reach adaptation than in gait adaptation. Finally, the duration of exercise $(\approx 5$ mins) in Charalambous et al. $(2018,2019)$ was shorter than that used here (30 minutes) and elsewhere (e.g., $>=15$ mins in Nepveu et al., 2017; Neva et al., 2019). A recent meta-analysis suggests that the nature of the motor task, and the timing and intensity of exercise are crucial factors in determining the efficacy of exercise in modulating motor learning (Wanner et al., 2020). Thus, careful consideration of these factors will be important for designing exercise-based interventions in movement rehabilitation.

A number of mechanisms might contribute to exercise-related improvements in learning. This improvement may be related to changes in the brain that accompany intense exercise, such as upregulation of neurotrophic factors such as brain derived neurotrophic factor, or proteins such as growth hormone, increased cortisol levels and/or changes to neurotransmitter release (Gligoroska \& Manchevska, 2012; Gray et al., 2013; Szuhany et al., 2015). In our exploratory analyses, we found a positive but non-significant relationship between performance improvements and BDNF increases in the treadmill condition. We note that our sample size might be underpowered to detect this effect, and larger sample sizes and/or more sensitive study designs (e.g., measuring plasma rather than serum BDNF) are required to robustly test the relationship between exerciseinduced changes in BDNF and adaptation (Szuhany et al., 2015; Dinoff et al., 2017). Other potential mediators for improved performance include the salience of the task at hand (i.e. how relevant/important is the task to the individual completing it) and the ability of 
the client to engage with and concentrate on the task. These two mediators (salience and concentration) are of particular importance, highlighted by stroke guideline statements that encourage active task practice outside of scheduled therapy hours to maximise functional return in rehabilitation (English, 2017).

Evidence supports the prescription of moderate to high intensity aerobic exercise to improve sensorimotor performance in healthy individuals. The present study extends these findings to demonstrate that in stroke survivors, utilising moderate to high intensity exercise can improve sensorimotor adaptation. A positive change in performance (measured as an increased adaptation) may represent an exercise induced change in the brain. It has been previously stated that BDNF concentrations increase following a single bout of aerobic exercise (Szuhany et al., 2015). BDNF is a neurotrophin that plays a key role in the formation of new neurons, development, and strengthening of existing neurons and in the restructure of the neuron pool with use (Lessmann \& Brigadski, 2009; Cunha et al., 2010; Hartmann et al., 2012). It may be possible to harness increased levels of BDNF following exercise to enhance sensorimotor performance. Increased levels of BDNF can occur in response to a program of aerobic exercise in neurological populations (Mackay et al., 2017). This is an important concept for chronic stroke survivors due to the stagnant nature and plateau that often accompanies this phase of recovery. Providing stroke survivors with a way to augment their recovery could lead to enhanced motor re-adaptation, functional gains and ultimately boost community participation of this group.

In this study, participants' average time post stroke was four years, representing the chronic phase of recovery. We note that although gains are still made in this phase of recovery, the magnitude of gains may be less than in a more acute recovery phase (Ballester et al., 2019). The majority of post-stroke recovery is generally observed to occur within the acute (1-7 days) and subacute phases of recovery (7 days -6 months) (Bernhardt et al., 2017). Harnessing potential changes in the brain due to exercise is important for all stages of recovery post stroke, but the magnitude and impact of this change may be increased in the earlier phases of recovery. It is therefore important to consider the timing of an aerobic exercise intervention post-stroke to maximise patient benefit in this way. Implementing an aerobic exercise intervention during the subacute phase of recovery may represent a more appropriate temporal window for enhancing neuroplastic benefit. Stroke survivors in the chronic phase of recovery, however should still be encouraged to participate in aerobic exercise for potential benefit.

\subsection{Limitations}

One possible limitation of this study was carryover effects. As this study was a within-subject (crossover) design, participants acted as their own control. Participants engaged in the sensorimotor adaptation task four times, on two separate testing days, with a oneweek washout period between the two testing days. Compared to the control intervention, the treadmill exercise intervention increased rapid error reduction performance from pre-to-post intervention. We controlled for carryover effects with a washout block with the perturbation removed at the end of the test session, as well as by employing an equal and opposite rotation on the second testing day in a counter-balanced order (30 degrees clockwise on Day 1 vs 30 degrees counter-clockwise on Day 2). We also randomised the number of participants who completed the control condition on their first visit compared to the treadmill condition.

The assessment item to quantify motor learning in this study was a sensorimotor adaptation task. Although this task has been widely used, it might not fully describe real-life motor skill acquisition (Krakauer et al., 2019). This sensorimotor adaptation task, however, enabled the sensitive detection of exercise-related improvements in motor learning here.

\section{Conclusions}

This is the first study to our knowledge to demonstrate an improvement in sensorimotor adaptation following moderate-high intensity exercise in a chronic stroke patient sample. Our findings are consistent with an increasing body of work demonstrating improved retention of other forms of motor learning with exercise, both in young healthy adults (Roig et al., 2012; Mang et al., 2014; Statton et al., 2015; Stavrinos \& Coxon, 2017), in stroke patients (Nepveu et al., 2017) and in Parkinson's disease (Steib et al., 2018; Wanner et al., 2021). Due to the neurological deficit incurred by stroke survivors, being able to improve adaptation of a motor task through aerobic exercise may have the potential to significantly improve function in this cohort. 


\section{Acknowledgments}

No funding was provided for this study. The authors would like to acknowledge the in kind contributions received from the School of Human Movement and Nutrition Science at The University of Queensland, and the School of Health and Rehabilitation Sciences at The University of Queensland in the form of utilisation of resources, building space and assistance from staff, including Katrina Kemp.

\section{Conflict of interest}

All of the authors wish to declare that they have no conflicts of interest in relation to this study.

\section{References}

Angulo-Barroso, R., Ferrer-Uris, B. \& Busquets, A. (2019) Enhancing Children's Motor Memory Retention Through Acute Intense Exercise: Effects of Different Exercise Durations. Frontiers in Psychology, 10, 2000.

Ballester, B.R., Maier, M., Duff, A., Cameirao, M., Bermudez, S., Duarte, E., Cuxart, A., Rodriguez, S., San Segundo Mozo, R.M. \& Verschure, P. (2019) A critical time window for recovery extends beyond one-year post-stroke. Journal of Neurophysiology, 122, 350-357.

Bernhardt, J., Hayward, K.S., Kwakkel, G., Ward, N.S., Wolf, S.L., Borschmann, K., Krakauer, J.W., Boyd, L.A., Carmichael, S.T., Corbett, D. \& Cramer, S.C. (2017) Agreed Definitions and a Shared Vision for New Standards in Stroke Recovery Research: The Stroke Recovery and Rehabilitation Roundtable Taskforce. Neurorehabilitation and Neural Repair, 31, 793-799.

Borg, G. (1982) Ratings of perceived exertion and heart rates during short-term cycle exercise and their use in a new cycling strength test. International Journal of Sports Medicine, 3, 153-158.

Brown, D.M.Y. \& Bray, S.R. (2018) Acute effects of continuous and high-intensity interval exercise on executive function. Journal of Applied Biobehavioral Research, 23, e12121.

Castellano, V. \& White, L.J. (2008) Serum brain-derived neurotrophic factor response to aerobic exercise in multiple sclerosis. Journal of the Neurological Sciences, 269, 85-91.

Chang, Y.K., Labban, J.D., Gapin, J.I. \& Etnier, J.L. (2012) The effects of acute exercise on cognitive performance: a metaanalysis. Brain Research, 1453, 87-101.

Charalambous, C.C., Alcantara, C.C., French, M.A., Li, X., Matt, K.S., Kim, H.E., Morton, S.M. \& Reisman, D.S. (2018) A single exercise bout and locomotor learning after stroke: physiological, behavioural, and computational outcomes. The Journal of Physiology, 596, 1999-2016.

Charalambous, C.C., French, M.A., Morton, S.M. \& Reisman, D.S. (2019) A single high-intensity exercise bout during early consolidation does not influence retention or relearning of sensorimotor locomotor long-term memories. Experimental Brain Research, 237, 2799-2810.

Cunha, C., Brambilla, R. \& Thomas, K.L. (2010) A simple role for BDNF in learning and memory? Frontiers in Molecular Neuroscience, 3,1 .

Dinoff, A., Herrmann, N., Swardfager, W. \& Lanctot, K.L. (2017) The effect of acute exercise on blood concentrations of brainderived neurotrophic factor in healthy adults: a meta-analysis. The European Journal of Neuroscience, 46, 1635-1646.

English, C. (2017) Clinical Guidelines for Stroke Management 2017, Melbourne Australia.

Ferrer-Uris, B., Busquets, A., Lopez-Alonso, V., FernandezDel-Olmo, M. \& Angulo-Barroso, R. (2017) Enhancing consolidation of a rotational visuomotor adaptation task through acute exercise. PLoS One, 12, e0175296.

Ghilardi, M.F., Gordon, J. \& Ghez, C. (1995) Learning a visuomotor transformation in a local area of work space produces directional biases in other areas. Journal of Neurophysiology, $73,2535-2539$

Gligoroska, J.P. \& Manchevska, S. (2012) The effect of physical activity on cognition - physiological mechanisms. Materia Socio-medica, 24, 198-202.

Globas, C., Becker, C., Cerny, J., Lam, J.M., Lindemann, U., Forrester, L.W., Macko, R.F. \& Luft, A.R. (2012) Chronic stroke survivors benefit from high-intensity aerobic treadmill exercise: a randomized control trial. Neurorehabilitation and Neural Repair, 26, 85-95.

Gray, J.D., Milner, T.A. \& McEwen, B.S. (2013) Dynamic plasticity: the role of glucocorticoids, brain-derived neurotrophic factor and other trophic factors. Neuroscience, 239, 214-227.

Hartmann, D., Drummond, J., Handberg, E., Ewell, S. \& PozzoMiller, L. (2012) Multiple approaches to investigate the transport and activity-dependent release of BDNF and their application in neurogenetic disorders. Neural Plasticity, 2012, 203734.

Heuer, H. \& Hegele, M. (2008) Constraints on visuo-motor adaptation depend on the type of visual feedback during practice. Experimental Brain Research, 185, 101-110.

Huberdeau, D.M., Haith, A.M. \& Krakauer, J.W. (2015) Formation of a long-term memory for visuomotor adaptation following only a few trials of practice. Journal of Neurophysiology, 114, 969-977.

Jordan, M. (1992) Forward models: Supervised learning with a distal teacher. Cognitive Science, 16, 307-354.

Kim, H.E., Parvin, D.E. \& Ivry, R.B. (2019) The influence of task outcome on implicit motor learning. eLife, 8, 363606.

Kitago, T. \& Krakauer, J.W. (2013) Motor learning principles for neurorehabilitation. Handbook of Clinical Neurology, 110, 93-103.

Krakauer, J.W., Ghez, C. \& Ghilardi, M.F. (2005) Adaptation to visuomotor transformations: consolidation, interference, and forgetting. The Journal of Neuroscience, 25, 473-478.

Krakauer, J.W., Hadjiosif, A.M., Xu, J., Wong, A.L. \& Haith, A.M. (2019) Motor Learning. Comprehensive Physiology, 9 , 613-663.

Kuys, S.S., Brauer, S.G. \& Ada, L. (2011) Higher-intensity treadmill walking during rehabilitation after stroke in feasible and not detrimental to walking pattern or quality: a pilot randomized trial. Clinical Rehabilitation, 25, 316-326. 
Lawrence, A. (2006) ACSM's guidelines for exercise testing and prescription. Lippincott Williams \& Wilkins, Baltimore.

Leow, L.A., Marinovic, W., de Rugy, A. \& Carroll, T.J. (2018) Task errors contribute to implicit aftereffects in sensorimotor adaptation. The European Journal of Neuroscience, 48, 33973409.

Leow, L.A., Marinovic, W., Riek, S. \& Carroll, T.J. (2017) Cerebellar anodal tDCS increases implicit learning when strategic re-aiming is suppressed in sensorimotor adaptation. PLoS One, 12, e0179977.

Lessmann, V. \& Brigadski, T. (2009) Mechanisms, locations, and kinetics of synaptic BDNF secretion: an update. Neuroscience Research, 65, 11-22.

MacKay-Lyons, M.J. \& Makrides, L. (2002) Cardiovascular stress during a contemporary stroke rehabilitation program: is the intensity adequate to induce a training effect? Archives of Physical Medicine and Rehabilitation, 83, 1378-1383.

Mackay-Lyons, M.J. \& Makrides, L. (2004) Longitudinal changes in exercise capacity after stroke. Archives of Physical Medicine and Rehabilitation, 85, 1608-1612.

Mackay, C.P., Kuys, S.S. \& Brauer, S.G. (2017) The Effect of Aerobic Exercise on Brain-Derived Neurotrophic Factor in People with Neurological Disorders: A Systematic Review and Meta-Analysis. Neural Plasticity, 2017, 4716197.

Mang, C.S., Snow, N.J., Campbell, K.L., Ross, C.J. \& Boyd, L.A. (2014) A single bout of high-intensity aerobic exercise facilitates response to paired associative stimulation and promotes sequence-specific implicit motor learning. Journal of Applied Physiology (1985), 117, 1325-1336.

Morehead, J.R. \& de Xivry, J.-J.O. (2021) A Synthesis of the Many Errors and Learning Processes of Visuomotor Adaptation. bioRxiv - The preprint server for biology.

Morehead, J.R., Qasim, S.E., Crossley, M.J. \& Ivry, R. (2015) Savings upon Re-Aiming in Visuomotor Adaptation. The Journal of Neuroscience, 35, 14386-14396.

Mutha, P.K., Sainburg, R.L. \& Haaland, K.Y. (2011) Critical neural substrates for correcting unexpected trajectory errors and learning from them. Brain, 134, 3647-3661.

Nepveu, J.F., Thiel, A., Tang, A., Fung, J., Lundbye-Jensen, J., Boyd, L.A. \& Roig, M. (2017) A Single Bout of HighIntensity Interval Training Improves Motor Skill Retention in Individuals With Stroke. Neurorehabilitation and Neural Repair, 31, 726-735.

Neva, J.L., Ma, J.A., Orsholits, D., Boisgontier, M.P. \& Boyd, L.A. (2019) The effects of acute exercise on visuomotor adaptation, learning, and inter-limb transfer. Experimental Brain Research, 237, 1109-1127.

Roemmich, R.T., Long, A.W. \& Bastian, A.J. (2016) Seeing the errors you feel enhances locomotor performance but not learning. Current biology, 26, 2707-2716.

Roig, M., Skriver, K., Lundbye-Jensen, J., Kiens, B. \& Nielsen, J.B. (2012) A single bout of exercise improves motor memory. PLoS One, 7, e44594.

Schaefer, S.Y., Haaland, K.Y. \& Sainburg, R.L. (2009) Dissociation of initial trajectory and final position errors during visuomotor adaptation following unilateral stroke. Brain Research, 1298, 78-91.
Schaefer, S.Y., Shelly, I.L. \& Thoroughman, K.A. (2012) Beside the point: motor adaptation without feedback-based error correction in task-irrelevant conditions. Journal of Neurophysiology, 107, 1247-1256.

Schulz, K.H., Gold, S.M., Witte, J., Bartsch, K., Lang, U.E., Hellweg, R., Reer, R., Braumann, K.M. \& Heesen, C. (2004) Impact of aerobic training on immune-endocrine parameters, neurotrophic factors, quality of life and coordinative function in multiple sclerosis. Journal of the Neurological Sciences, $225,11-18$.

Seidler, R.D. \& Carson, R.G. (2017) Sensorimotor Learning: Neurocognitive Mechanisms and Individual Differences. Journal of Neuroengineering and Rehabilitation, 14, 74.

Statton, M.A., Encarnacion, M., Celnik, P. \& Bastian, A.J. (2015) A Single Bout of Moderate Aerobic Exercise Improves Motor Skill Acquisition. PLoS One, 10, e0141393.

Stavrinos, E.L. \& Coxon, J.P. (2017) High-intensity Interval Exercise Promotes Motor Cortex Disinhibition and Early Motor Skill Consolidation. Journal of Cognitive Neuroscience, 29, 593-604.

Steib, S., Wanner, P., Adler, W., Winkler, J., Klucken, J. \& Pfeifer, K. (2018) A Single Bout of Aerobic Exercise Improves Motor Skill Consolidation in Parkinson's Disease. Frontiers in Aging Neuroscience, 10, 328.

Stinear, C.M., Lang, C.E., Zeiler, S. \& Byblow, W.D. (2020) Advances and challenges in stroke rehabilitation. The Lancet Neurology, 19, 348-360.

Szuhany, K.L., Bugatti, M. \& Otto, M.W. (2015) A meta-analytic review of the effects of exercise on brain-derived neurotrophic factor. Journal of Psychiatric Research, 60, 56-64.

Taylor, J.A., Krakauer, J.W. \& Ivry, R.B. (2014) Explicit and implicit contributions to learning in a sensorimotor adaptation task. The Journal of Neuroscience, 34, 3023-3032.

Thomas, R., Johnsen, L.K., Geertsen, S.S., Christiansen, L., Ritz, C., Roig, M. \& Lundbye-Jensen, J. (2016) Acute Exercise and Motor Memory Consolidation: The Role of Exercise Intensity. PLoS One, 11, e0159589.

Tseng, Y.W., Diedrichsen, J., Krakauer, J.W., Shadmehr, R. \& Bastian, A.J. (2007) Sensory prediction errors drive cerebellum-dependent adaptation of reaching. Journal of Neurophysiology, 98, 54-62.

Vickers, A.J. (2001) The use of percentage change from baseline as an outcome in a controlled trial is statistically inefficient: a simulation study. BMC Medical Research Methodology, 1,6 .

Wanner, P., Cheng, F.H. \& Steib, S. (2020) Effects of acute cardiovascular exercise on motor memory encoding and consolidation: A systematic review with meta-analysis. Neuroscience and Biobehavioural Reviews, 116, 365-381.

Wanner, P., Winterholler, M., Gassner, H., Winkler, J., Klucken, J., Pfeifer, K. \& Steib, S. (2021) Acute exercise following skill practice promotes motor memory consolidation in Parkinson's disease. Neurobiology of Learning and Memory, 178, 107366 .

Welch, R.B. (1969) Adaptation to prism-displaced vision: The importance of target-pointing. Perception \& Psychophysics, $5,305-309$. 